\title{
SYNTHESIS AND STABILITY OF RESVERATROL-GOLD NANOPARTICLE-POLYETHYLENE GLYCOL-FOLIC ACID CONJUGATES
}

\author{
SUTRIYO SUTRIYO*, RADITYA ISWANDANA, FIRDA MARETHA IVARIANI \\ Laboratory of Pharmaceutics and Pharmaceutical Technology, Faculty of Pharmacy, Universitas Indonesia, Depok, 16424, Indonesia. \\ Email: sutriyo@farmasi.ui.ac.id
}

Received: 26 September 2019, Revised and Accepted: 17 December 2019

\section{ABSTRACT}

Objective: Gold nanoparticles (AuNPs) can be used as targeted drug delivery systems, however, AuNPs have high surface energy and easily aggregate, thus negatively impacting nanoparticle stability. Therefore, it is necessary to add a stabilizing agent to AuNPs. To synthesize AuNPs stabilized by polyethylene glycol conjugated to folic acid (FA), thus creating a model drug (resveratrol [RSV]) carrier that targets FA receptors on cancer cells.

Methods: AuNPs were synthesized using the Turkevich method and stabilized by adding FA conjugated to polyethylene glycol (PEG). After RSV was loaded, the conjugate was physically characterized and subjected to stability tests.

Results: The RSV-AuNP had an average particle size of $51.97 \mathrm{~nm}$ (polydispersity index [PDI] 0.694, zeta potential - 24.6 mV). The RSV-AuNP-PEG-FA conjugate (RSV-AuNP-PEG-FA) had an average particle size of $195.6 \mathrm{~nm}(\mathrm{PDI}=0.233$, zeta potential=-21.1 mV). Stability tests showed that RSV-AuNPPEG-FA was more stable than RSV-AuNP. Furthermore, RSV-AuNP-PEG-FA and RSV-AuNP were more stable in buffer pH 7.4 and bovine serum albumin $2 \%$ than in buffer $\mathrm{pH} 4$, cysteine $1 \%$, and $\mathrm{NaCl} 0.9 \%$ solutions.

Conclusion: PEG-FA conjugates can improve the stability of RSV-loaded AuNP.

Keywords: Polyethylene glycol, Folic acid, Gold nanoparticle, Resveratrol, Stability.

(C) 2020 The Authors. Published by Innovare Academic Sciences Pvt Ltd. This is an open access article under the CC BY license (http://creativecommons. org/licenses/by/4. 0/) DOI: http://dx.doi.org/10.22159/ijap.2020.v12s1.FF051

\section{INTRODUCTION}

Nanoparticles have several advantages, such as improvement in the intracellular infiltration, circulation time of the drug, and enhanced hydrophobic solubility [1]. They consist of different biodegradable materials such as natural or synthetic polymers, lipids, or metals [2]. Gold nanoparticles (AuNPs) are some of the most widely employed nanoparticles in medicine. AuNPs have been extensively applied in the fields of general drug delivery, targeted drug delivery, and disease diagnosis [3]. While AuNPs are very effective at targeted drug delivery, they have high surface energy and easily aggregate [4]. Stabilization of AuNPs can be accomplished by employing surfactants, by adding functional groups to the nanoparticle surface, or coating the nanoparticle with a polymer layer. One polymer that can be used as a stabilizing agent is polyethylene glycol (PEG). PEG can be used to coat nanoparticles, thus increasing nanoparticle stability and preventing nanoparticle aggregation [4]. PEGylation of nanoparticles can improve their in vivo stability, prevent surface attachment of opsonin proteins, and increase nanoparticle accumulation at tumor sites to increase antitumor activity [5].

Other important factors exist for increasing the cellular uptake process, such as nanoparticle surface modification with targeting moieties. Active targeting is a strategy, where functional targeting moieties are peripherally conjugated to the nanoparticle system, thus enabling the preferential accumulation of particles at the tumor site. In active targeting-based cancer treatments, common molecular targets include carbohydrates, antibodies, and cell surface receptors [6]. Folate receptors are present in many cancer cells [7], such as colon, lung, prostate, ovary, breast, and brain cancer cells [8]. Therefore, targeted treatment at folate receptors can be an effective option for cancer therapy. Nanoparticles conjugated with folic acid (FA) can selectively bind to cell surface folate receptors on cancerous cells and be internalized through endocytosis [6]. Since FA has a very high affinity for folate receptors, FA is a suitable ligand for targeted therapeutic receptors $[9,10]$.

Resveratrol (RSV) (trans-3,4,5-trihydroxystilbene) is a naturally occurring polyphenol compound that possesses a variety of biological activities, such as anticancer and antioxidant activity [11-14]. Sun Young Park (2016) conducted a study employing RSV-conjugated AuNPs for breast cancer cell therapy and found that RSV acted as both a reducing agent and stabilizer for the AuNPs.

The purpose of this study was to synthesize a site-targeting drug delivery system using AuNPs stabilized by the addition of PEG and conjugated with FA-RSV which was used as a model drug compound, PEG was employed as a nanoparticle stabilizing agent, and FA was the cell-targeting ligand.

\section{METHODS}

\section{Materials}

All materials were used as received: RSV standard (Sigma-Aldrich, USA), $\mathrm{HAuCl}_{4}$ (Sigma-Aldrich, USA), PEG-bis-amine (Sigma-Aldrich, USA), FA (Sigma-Aldrich, USA), sodium citrate (Sigma-Aldrich, USA), phosphatebuffered saline (Sigma-Aldrich, USA), bovine serum albumin (BSA) (Sigma-Aldrich, USA), cysteine (Sigma-Aldrich, USA), sodium chloride (Merck, Germany), $N$-(3-dimethylaminopropyl)- $N$-ethylcarbodiimide (Sigma-Aldrich, USA), $N$-hydroxysuccinimide (Sigma-Aldrich, USA), hydrochloric acid (Merck, Germany), methanol (Merck, Germany), ethanol (Merck, Germany), acetonitrile (Merck, Germany), distilled water, and Aqua Bidest (Ika Pharmindo, Indonesia).

\section{Methods}

Synthesis of PEG-FA

FA (FA; $25 \mathrm{mg}$ ) was conjugated to PEG-bis-amine (250 mg; 1.5:1.0 molar ratio) in the presence of EDC ( $300 \mathrm{mg})$ and NHS $(100 \mathrm{mg})$. The reaction 
was accelerated by adding $\mathrm{HCl}(2 \%$ in water, $0.5 \mathrm{ml})$ and stirred for $5 \mathrm{~h}$ at room temperature. The reaction mixture was then centrifugated, and the top layer, containing FA conjugated to PEG (PEG-FA), was freezedried to obtain the powder of PEG-FA conjugates.

\section{Synthesis of the RSV-AuNP-PEG-FA conjugate (RSV-AuNP-PEG-FA)}

AuNPs were synthesized according to the Turkevich method by reducing a hydrogen tetrachloroaurate solution using sodium citrate. A sodium citrate solution $(0.705 \mathrm{~mL}$ and $130 \mathrm{mM})$ was added to a solution of $\mathrm{HAuCl}_{4} \cdot 3 \mathrm{H}_{2} \mathrm{O}(25 \mathrm{~mL}$ and $1 \mathrm{mM})$ heated to approximately $95^{\circ} \mathrm{C}$ with stirring. The color of the solution changed instantly from pale yellow to colorless. Then, the solution changed to dark blue and then to a deep red burgundy after a few minutes. Stirring and heating of the solution were maintained for several minutes after the addition of sodium citrate, and the solution was stirred during cooling at room temperature. PEG-FA ( $250 \mathrm{mg}$ ) was added to the above AuNP $(25 \mathrm{ml})$ solution. The mixture was stirred at room temperature for $4 \mathrm{~h}$ to obtain AuNP-PEG-FA. Unreacted PEG-FA was removed by centrifugation. RSV solution $(5.7 \mathrm{~mL}, 10 \mu \mathrm{g} / \mathrm{mL})$ was added drop by drop to the continuously stirred AuNP-PEG-FA solution (150 rpm for $2 \mathrm{~h}$ at room temperature) to obtain RSV-AuNP-PEG-FA.

\section{Synthesis of RSV-AuNP conjugates without stabilizer (RSV-AuNP)}

AuNPs were synthesized according to the Turkevich method by reducing a hydrogen tetrachloroaurate solution using sodium citrate as above. RSV solution ( $5.7 \mathrm{~mL}, 10 \mu \mathrm{g} / \mathrm{mL}$ ) was added drop by drop to the continuously stirred AuNP solution (150 rpm for $2 \mathrm{~h}$ at room temperature) to obtain RSV-AuNP.

\section{Characterization of RSV-AuNP and RSV-AuNP-PEG-FA}

General observations of RSV-AuNP and RSV-AuNP-PEG-FA

Observations of RSV-AuNP and RSV-AuNP-PEG-FA were recorded qualitatively from visual inspection of the conjugates.

\section{Absorbance and wavelength measurements}

The absorbance and wavelength measurements of RSV-AuNP-PEG-FA were performed using an ultraviolet (UV)-1800 spectrophotometer (Shimadzu, Japan) in the wavelength range of 400-800 nm.

\section{Infrared spectrum analysis}

The Fourier-transform infrared (FTIR) spectra (KBr pellet) of RSV, PEG-FA, AuNP, RSV-AuNP, and RSV-AuNP-PEG-FA were recorded on FTIR-8400S spectrometer (Shimadzu, Japan) in a wavenumber region of $400-4000 \mathrm{~cm}^{-1}$

\section{Particle size and size distribution measurements}

The average particle size (z-average) and polydispersity index (PDI) of RSV-AuNP and RSV-AuNP-PEG-FA were determined through dynamic light scattering (DLS) at $25^{\circ} \mathrm{C}$ using a Malvern Zetasizer (Nano ZS, Malvern Instruments, Worcestershire, UK).

\section{Zeta potential measurements}

The zeta potential of RSV-AuNP and RSV-AuNP-PEG-FA was measured using a Malvern Zetasizer at $25^{\circ} \mathrm{C}$ (Nano ZS, Malvern Instruments, Worcestershire, UK).

\section{Determination of RSV entrapment efficiency (\%EE)}

A $1000 \mathrm{ppm}$ RSV solution in methanol $(1 \mathrm{mg} / \mathrm{mL})$ was serially diluted $(1,5,10,15,20$, and $25 \mathrm{ppm})$ to obtain a calibration curve. RSV-AuNPPEG-FA solution $(2 \mathrm{~mL})$ was ultracentrifuged $\left(12,000 \mathrm{rpm}\right.$ at $\left.27^{\circ} \mathrm{C}\right)$ for $1 \mathrm{~h}$, and the supernatant was syringe filtered $(0.22 \mu \mathrm{m}$ pore size filter) and analyzed by HPLC. The amount of RSV trapped in the RSVAuNP-PEG-FA was determined indirectly using HPLC by subtracting the quantity in the supernatant from the total amount added. These analyses were performed in triplicate. The \%EE of RSV was calculated using the following equation:

$$
\% \mathrm{EE}=\frac{\mathrm{C}_{\text {total }}-\mathrm{C}_{\text {free }}}{\mathrm{C}_{\text {total }}} \times 100 \%
$$

\section{Stability test of RSV-AuNP and RSV-AuNP-PEG-FA}

The stability test of RSV-AuNP and RSV-AuNP-PEG-FA was performed using $4 \mathrm{~mL}$ of each sample equally aliquoted into $2 \mathrm{~mL}$ each of buffer $\mathrm{pH} 4$, buffer $\mathrm{pH} 7.4$, BSA $2 \%$, cysteine $1 \%$, and $\mathrm{NaCl} 0.9 \%$. The physical appearance (aggregation), absorbance, changes in maximum absorbance wavelength, and changes in the full width at half maximum value were evaluated over 4 weeks. In the $1^{\text {st }}$ week, evaluations were performed daily. Thereafter, evaluations were performed every 3 days.

\section{RESULTS AND DISCUSSION}

\section{Synthesis of RSV-AuNP-PEG-FA}

A brief optimization of the reaction time for AuNP synthesis after the addition of sodium citrate was performed (Table 1). As shown, an increase in absorbance was observed from 5 to $15 \mathrm{~min}$. However, when the reaction time was increased to $25 \mathrm{~min}$, the absorbance decreased to 0.305 . The decrease in absorbance indicated aggregate formation. Therefore, $15 \mathrm{~min}$ of heating and stirring after sodium citrate addition was deemed optimal, as was also reported in the literature $[15,16]$.

\section{Characterization of RSV-AuNP and RSV-AuNP-PEG-FA}

General observations of RSV-AuNP and RSV-AuNP-PEG-FA

The RSV-AuNP-PEG-FA solution was observed to have a red burgundy color. A red burgundy solution is characteristic of non-aggregated nanoparticles due to a rather narrow absorption wavelength at $520 \mathrm{~nm}$. When the solution becomes blue, a AuNP solution is indicated to be partially aggregated [17]. The RSV-AuNP-PEG-FA has a clearer red burgundy color than RSV-AuNP. The RSV-AuNP-PEG-FA has a relatively stable red burgundy color after 7 days, while the RSV-AuNP became more purple by the $3^{\text {rd }}$ day. Thus, the PEG coating was observed to improve nanoparticle stability to prevent AuNP aggregation [4].

\section{Absorbance and wavelength measurements}

Fig. 1 presents the UV-visible absorbance spectra of PEG-FA (0.256 at $345.40 \mathrm{~nm}$ and 1.065 at $280.80 \mathrm{~nm}$ ), AuNP (0.404 at $528 \mathrm{~nm}$ ), RSVAuNP (0.260 at $529 \mathrm{~nm}$ ), and RSV-AuNP-PEG-FA (0.890 at $533 \mathrm{~nm}$ and 1.989 at $279 \mathrm{~nm}$ ). As shown in Fig. 1, the RSV-AuNP-PEG-FA curve was sharper than RSV-AuNP. According to Turkevich, the increase in particle size can result in a marked change in the width of spectral. It is possible that the particle size of RSV-AuNP-PEG-FA was smaller than that of RSV-AuNP. Moreover, in RSV-AuNP, there was no absorbance at $279 \mathrm{~nm}$, whereas in RSV-AuNP-PEG-FA, there was an absorbance at $279 \mathrm{~nm}$, indicative of a successful conjugation between PEG-FA and AuNP.

Table 1: Optimization of reaction time after sodium citrate addition

\begin{tabular}{llll}
\hline No. & $\begin{array}{l}\text { Duration of stirring } \\
\text { and warming (min) }\end{array}$ & $\boldsymbol{\lambda}(\mathbf{n m})$ & Absorbance \\
\hline 1. & 5 & 528.00 & 0.404 \\
2. & 15 & 528.50 & 0.480 \\
3. & 25 & 538.50 & 0.305 \\
\hline
\end{tabular}

Table 2: Selected parameters for RSV-AuNP and RSV-AuNP-PEG-FA

\begin{tabular}{lll}
\hline & RSV-AuNP & RSV-AuNP-PEG-FA \\
\hline Particle size $(\mathrm{nm})$ & 51.97 & 195.6 \\
PDI & 0.694 & 0.233 \\
Zeta potential $(\mathrm{mV})$ & -24.6 & -21.1 \\
\hline
\end{tabular}

AuNP: Gold nanoparticle, RSV: Resveratrol, PDI: Polydispersity, FA: Folic acid PEG: Polyethylene glycol 


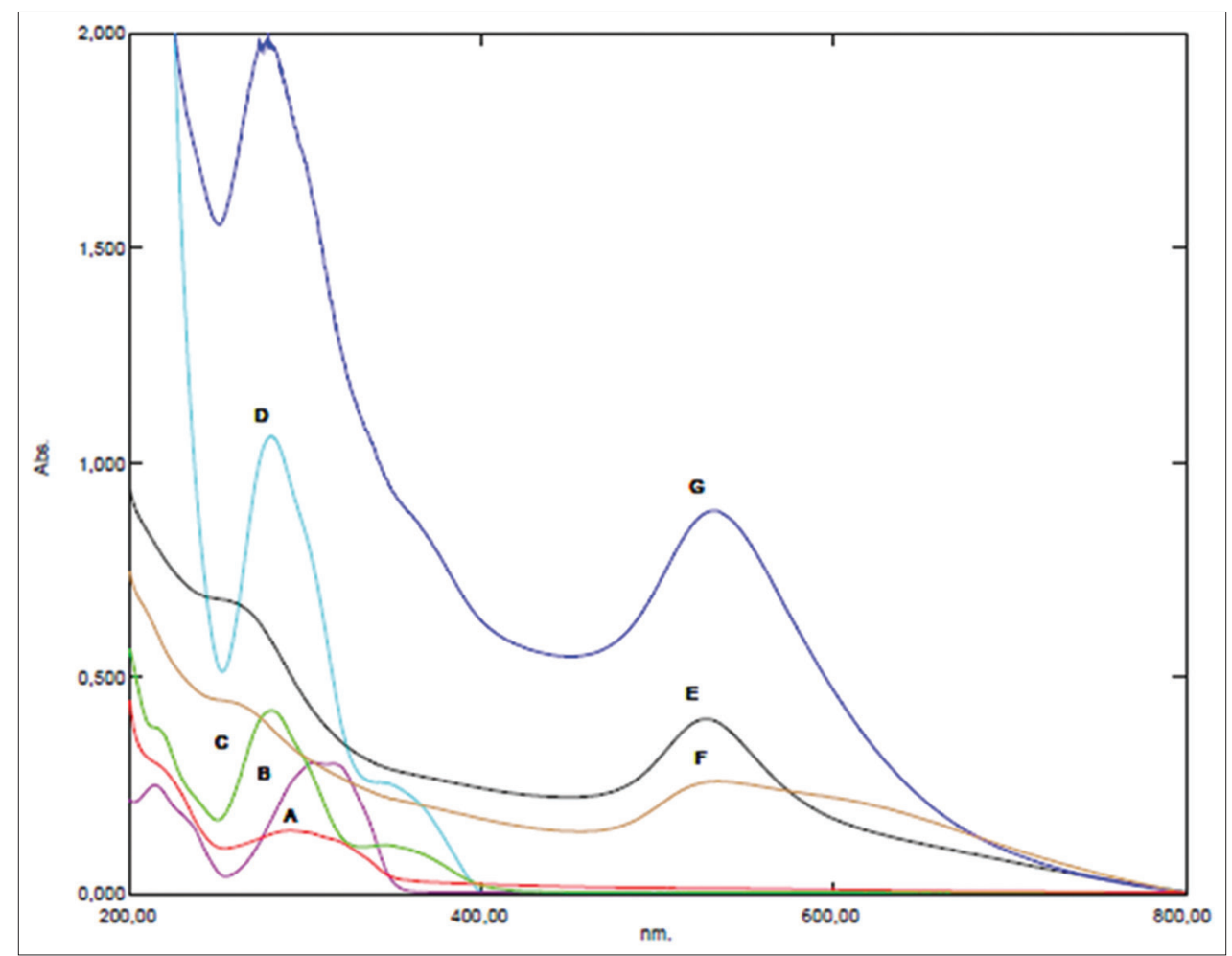

Fig. 1: Ultraviolet-visible spectra of polyethylene glycol (PEG)-bis-amine (a), resveratrol (RSV) (b), folic acid (FA) (c), PEG-FA (d), gold nanoparticle (AuNP) (e), RSV-AuNP (f), and RSV-AuNP-PEG-FA (g)

\section{Infrared spectrum analysis}

Fig. 2 presents the FTIR spectra of RSV, PEG-FA, AuNP, RSV-AuNP, and RSV-AuNP-PEG-FA. The characteristic peaks of RSV-AuNP-PEG-FA are 3444.96 and $3284.88 \mathrm{~cm}^{-1}(\mathrm{OH}), 1728.28 \mathrm{~cm}^{-1}(\mathrm{C}=0), 1647.26 \mathrm{~cm}^{-1}$ $(\mathrm{C}=\mathrm{C}), 1546.96$ and $1512.24 \mathrm{~cm}^{-1}$ ( $\mathrm{C}=\mathrm{C}$ aromatic), and $2357.09 \mathrm{~cm}^{-1}$. The same functional groups present on PEG-FA and RSV-AuNP-PEG-FA, such as $\mathrm{O}-\mathrm{H}, \mathrm{C}=\mathrm{O}$, and $\mathrm{C}=\mathrm{C}$, indicated that PEG-FA could be found on RSV-AuNP-PEG-FA. The peak intensity at $2357.09 \mathrm{~cm}^{-1}$ in RSV-AuNPPEG-FA was stronger than that of PEG-FA, AuNP, and RSV-AuNP because the PEG-FA and AuNP bond add to this peak. In contrast, the peak intensity of RSV at $833.28 \mathrm{~cm}^{-1}$ (para-substituted) disappeared in RSVAuNP-PEG-FA, indicative of RSV being absorbed into the AuNP.

\section{Particle size and size distribution measurements}

The particle size, particle size distribution, and PDI values of RSVAuNP-PEG-FA were obtained using a particle size analyzer with the DLS method. The DLS inner diameter is a value that refers to how the particle diffuses in a liquid and is referred to as the hydrodynamic diameter. This diameter is the spherical diameter and has the same translation diffusion coefficient as the particle [18]. The translation diffusion coefficient depends not only on the core particle size but also on each surface structure [19]. The average particle size formed from RSV-AuNP-PEG-FA was $195.6 \mathrm{~nm}$, while that of RSV-AuNP was $51.97 \mathrm{~nm}$. This difference in size results from PEG coating the surface of the AuNP. The particle-size distribution values based on the volume of the RSVAuNP-PEG-FA show that as many as $10 \%$ of the particles formed were $<19.0 \mathrm{~nm}, 50 \%$ of the particles formed were $<26.4 \mathrm{~nm}$, and $90 \%$ of the particles formed were $<88.1 \mathrm{~nm}$. PDI is a value to determine the particlesize distribution and ranges from 0 to 1 . If the PDI value is $<0.7$, then the system is considered monodispersed. However, if the PDI value is $>0.7$, then the system is considered polydisperse [20]. The PDI value of RSV-AuNP-PEG-FA was 0.233; therefore, this is a monodispersed system.

Zeta potential measurements

The zeta potential of RSV-AuNP-PEG-FA was $-21.1 \mathrm{mV}$, while that of RSV-AuNP was $-24.6 \mathrm{mV}$. According to the American Filtration and Separations Society, RSV-AuNP-PEG-FA belongs within the "fine dispersion threshold." The zeta potential value of RSV-AuNP-PEGFA was smaller than that of RSV-AuNP. This can be attributed to PEGylation. PEGylation is known to reduce the zeta potential value in accordance with the theory of steric stability in nanoparticles [8]. By surrounding the metal center with a steric material, a repulsive force is created between the metal nanoparticles [21]. Table 2 shows the comparison of RSV-AuNPs conjugate parameters with RSV-AuNPsPEG-FA conjugates.

\section{Determination of RSV\%EE}

The calibration curve equation was $y=92620 \quad x-28703$ with a correlation coefficient value $r=0.997$. The retention time of the RSV in RSV-AuNP-PEG-FA was $15.181 \mathrm{~min}$, while in the standard solution, this was $15.396 \mathrm{~min}$. The amount of RSV trapped in the nanoparticles (analysis in triplicate) was $57.67 \%$ (average of $57.74 \%, 57.36 \%$, and $57.90 \%$ ). These results show that RSV was entrapped in RSV-AuNPPEG-FA.

\section{Stability tests of RSV-AuNP and RSV-AuNP-PEG-FA}

The results of the stability test showed that RSV-AuNP-PEG-FA was more stable than RSV-AuNP (Fig. 3). This was expected as PEG can improve the stability of nanoparticles according to the steric stability theory (shear dispersion around the metal center by steric materials) [21]. In buffer $\mathrm{pH}$ 4, both RSV-AuNP and RSV-AuNP-PEG-FA were not stable because changes in $\mathrm{pH}$ change can cause AuNPs to be aggregate. The acidic $\mathrm{pH}$ four buffer medium was intended to simulate the condition of cancer cells. In the RSV-AuNP-PEG-FA drug delivery system, FA would interact with folate receptors expressed on the cancer cell surface. After endocytosis of the nanoparticle into an acidic environment, the model drug (RSV) could be released from nanoparticle destabilizations. In medium buffer pH 7.4 and BSA 2\%, both RSV-AuNP and RSV-AuNP-PEGFA were stable. Buffer $\mathrm{pH} 7.4$ has an osmolarity and ion concentration isotonic or equivalent to the condition of human body fluids, and BSA $2 \%$ simulated protein in plasma (human plasma can contain up to $60 \%$ total protein). Stability in these media suggests that the nanoparticle 


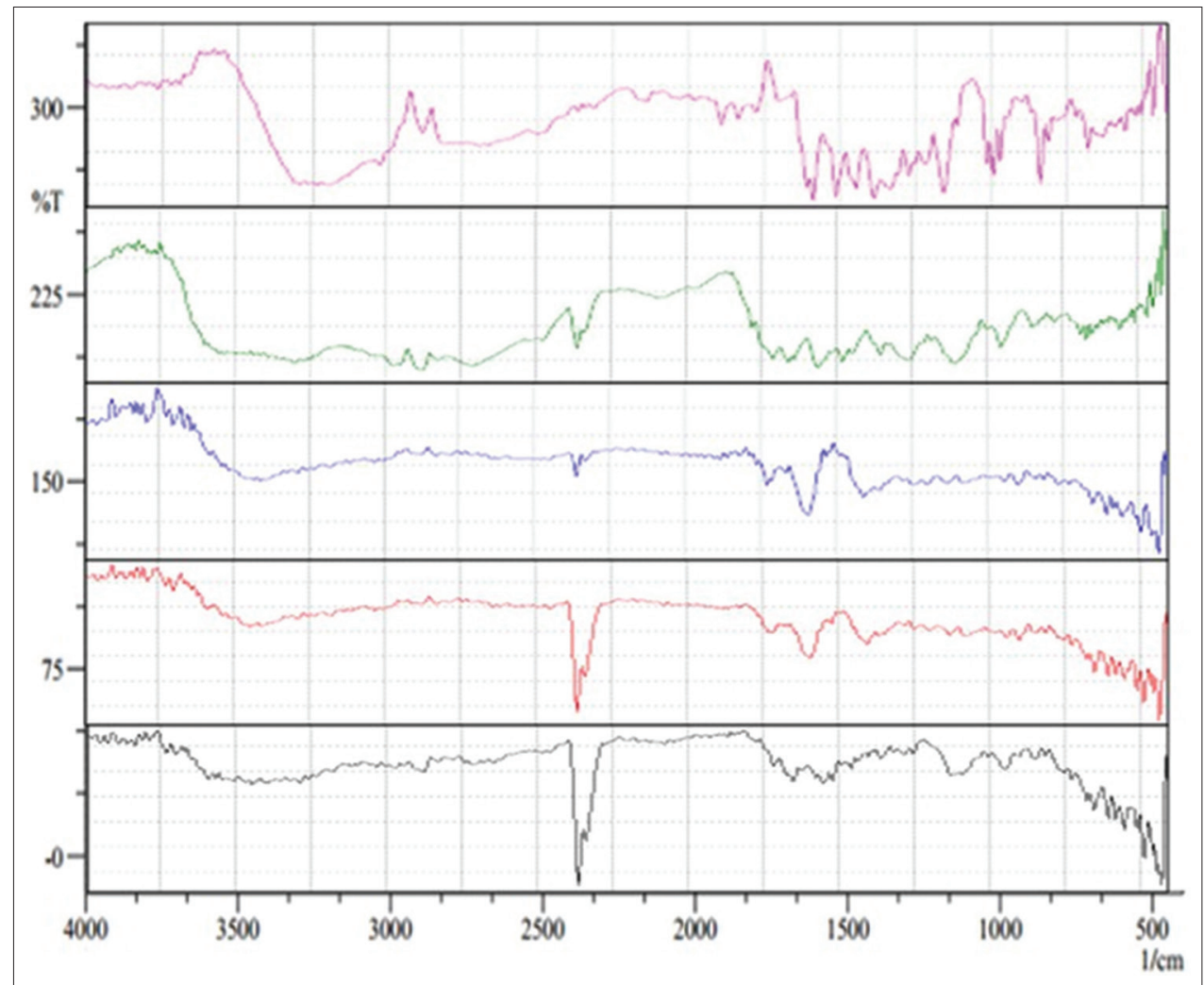

Fig. 2: Fourier-transform infrared spectra of resveratrol (RSV) (purple), polyethylene glycol (PEG)-folic acid (FA) (green), gold nanoparticle (AuNP) (blue), RSV-AuNP (red), and RSV-AuNP-PEG-FA (black)
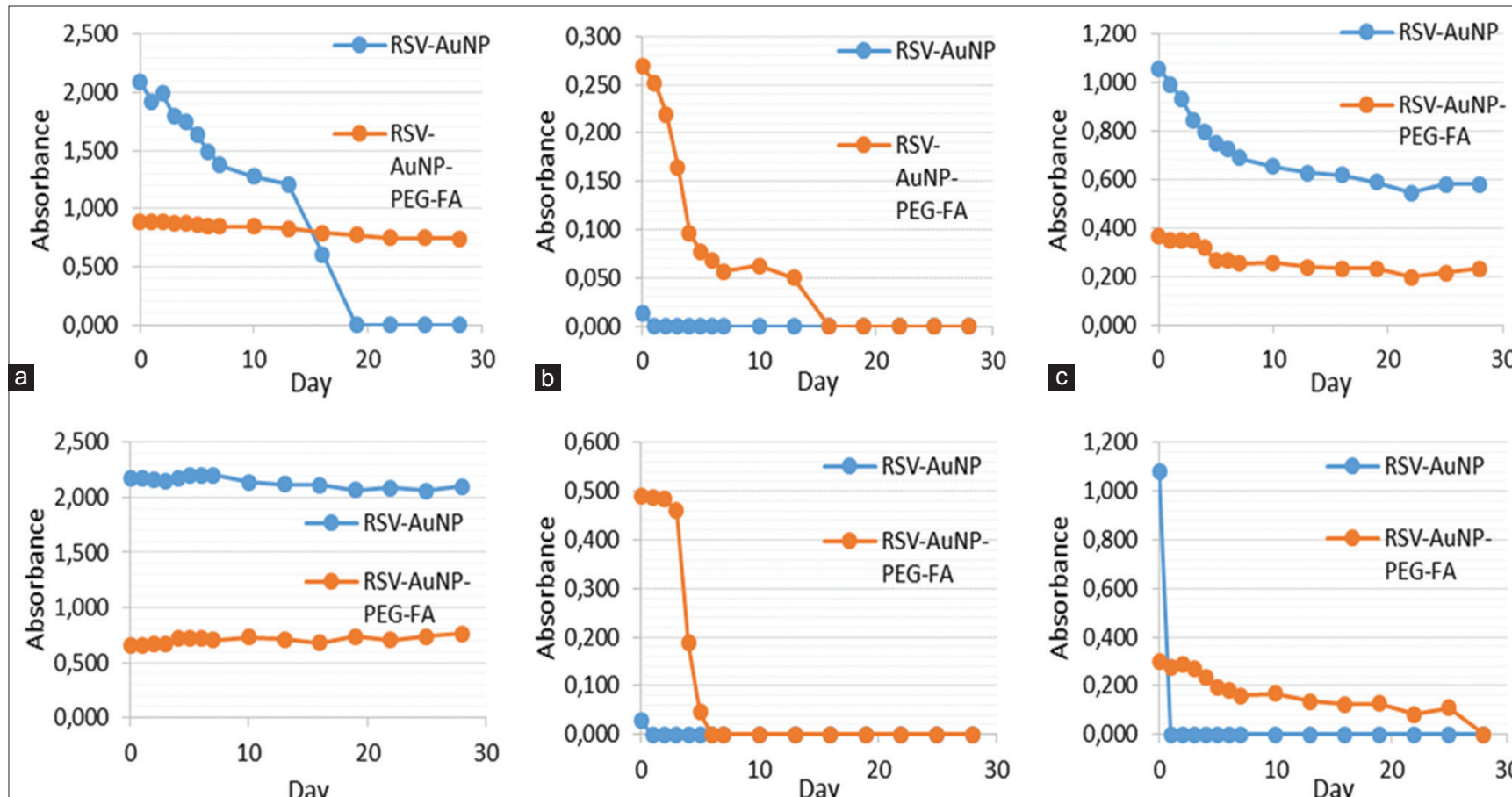

d
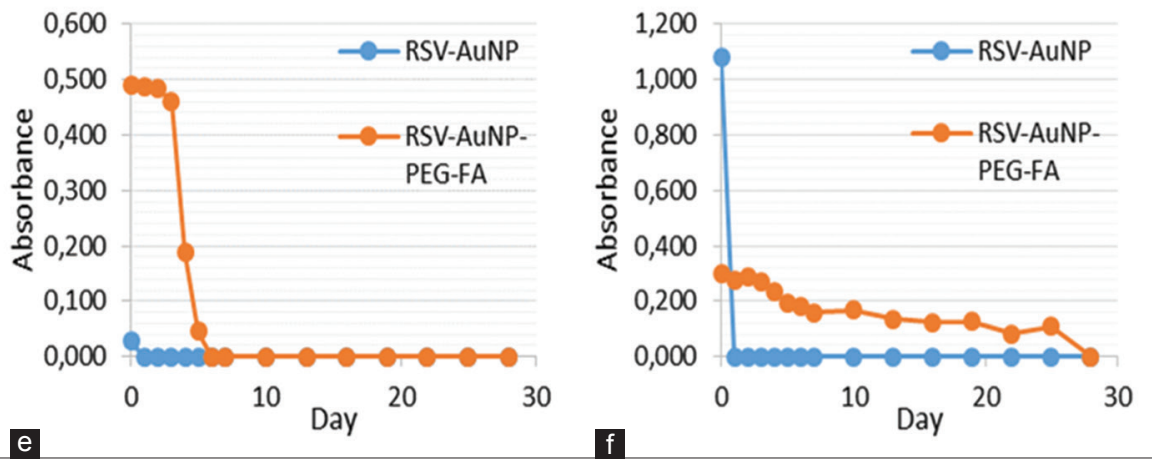

Fig. 3: Stability tests of resveratrol (RSV)-gold nanoparticle (AuNP) and RSV-AuNP-polyethylene glycol-folic acid based on absorbance in (a) without medium, (b) buffer $\mathrm{pH} 4$, (c) buffer $\mathrm{pH} \mathrm{7.4,} \mathrm{(d)} \mathrm{bovine} \mathrm{serum} \mathrm{albumin} \mathrm{2 \% ,} \mathrm{(e)} \mathrm{cysteine} \mathrm{1 \% ,} \mathrm{and} \mathrm{(f)} \mathrm{NaCl} 0.9 \%$

drug delivery system could stay intact in human plasma for several weeks and extend the drug release period. In cysteine $1 \%$ and $\mathrm{NaCl}$ $0.9 \%$, both RSV-AuNP and RSV-AuNP-PEG-FA were not stable (Fig. 3). The thiol groups in cysteine $1 \%$ interact with the gold and cause nanoparticle aggregation after breaking through the PEG coat. In $\mathrm{NaCl}$ $0.9 \%$, the PEG coat could not protect against the presence of additional electrolytes (cations and anions); moreover, aggregation to larger particles was observed [22]. 


\section{CONCLUSION}

RSV-AuNP-PEG-FA was successfully synthesized. The resulting particle solution was red burgundy in color and the average particle size was $195.6 \mathrm{~nm}(\mathrm{PDI}=0.233$, zeta potential $=-21.1 \mathrm{mV})$. The $\% \mathrm{EE}$ of RSV in RSV-AuNP-PEG-FA was $57.67 \%$. Stability tests showed that RSV-AuNPPEG-FA was more stable than RSV-AuNP and the stability of RSV-AuNPPEG-FA and RSV-AuNP in buffer pH 7.4 and BSA $2 \%$ was better than that in buffer $\mathrm{pH} 4$, cysteine $1 \%$, and $\mathrm{NaCl} 0.9 \%$. From this research, we can conclude that the stabilization imparted by PEG-FA to AuNPs can improve the overall stability of the RSV-AuNP drug delivery system.

\section{ACKNOWLEDGMENT}

We gratefully acknowledge the funding from Directorate of Research and Community Engagement Universitas Indonesia, via Hibah PITTA 2018 supporting this work.

\section{CONFLICTS OF INTEREST}

The authors declare that there are no conflicts of interest.

\section{REFERENCES}

1. Kadian, R. Nanoparticles: A promising drug delivery approach. Asian J Pharm Clin Res 2018;11:30-5.

2. Lavanya N, Muzib I, Jithan A, Umamahesh B. Novel nanoparticles for the oral delivery of low molecular weight heparin: In vitro and in vivo assessment. Asian J Pharm Clin Res 2017;10:254-61.

3. Khan A, Rashid R, Murtaza G, Zahra A. Nanoparticles: Synthesis and applications in drug delivery. Trop J Pharm Res 2014;13:1169-77.

4. Rahme K, Chen L, Hobbs R, Morris M, O'Driscoll C, Holmes J. PEGylated gold nanoparticles: Polymer quantification as a function of PEG lengths and nanoparticle dimensions. RSC Adv 2013;3:6085-94.

5. Artini I, Gusti A. The role of nanoparticles in cancer management in the era of targeting therapy [Peranan nanopartikel dalam penatalaksanaan kanker di era targeting therapy]. Indones J Cancer 2013;7:111-7

6. Sadhasivam S, Savitha S, Wu CJ, Lin FH, Stobiński L. Carbon encapsulated iron oxide nanoparticles surface engineered with polyethylene glycol-folic acid to induce selective hyperthermia in folate over expressed cancer cells. Int J Pharm 2015:480:8-14.

7. Parker N, Turk MJ, Westrick E, Lewis JD, Low PS, Leamon CP. Folate receptor expression in carcinomas and normal tissues determined by a quantitative radioligand binding assay. Anal Biochem 2005;338:284-93.

8. Hou Z, Zhan C, Jiang Q, Hu Q, Li L, Chang D, et al. Both FA and mPEG-conjugated chitosan nanoparticles for targeted cellular uptake and enhanced tumor tissue distribution. Nanoscale Res Lett 2011;6:563.

9. Low PS, Antony AC. Folate receptor-targeted drugs for cancer and inflammatory diseases. Adv Drug Deliv Rev 2004;56:1055-8.

10. Dhawan D, Ramos-Vara JA, Naughton JF, Cheng L, Low PS, Rothenbuhler R, et al. Targeting folate receptors to treat invasive urinary bladder cancer. Cancer Res 2013;73:875-84.

11. Mulakayala C, Babajan B, Madhusudana P, Anuradha CM, Rao RM, Nune RP, et al. Synthesis and evaluation of resveratrol derivatives as new chemical entities for cancer. J Mol Graph Model 2013;41:43-54.

12. Simon C, Britton RG, Cai H, Gescher AJ, Brown K, dan Jenkins PR. Novel analogues of resveratrol: Metabolism and inhibition of colon cancer cell proliferation. Tetrahedron 2013;69:6203-12.

13. He S, Yan X. From resveratrol to its derivatives: New sources of natural antioxidant. Curr Med Chem 2013;20:1005-17.

14. Frombauma M, Clanche SL, Bonnefont-Rousselot D, dan Borderie D. Antioxidant effects of resveratrol and other stilbene derivatives on oxidative stress and NO bioavailability: Potential benefits to cardiovascular diseases. Biochimie 2012;94:169-76.

15. Kimling J, Maier M, Okenve B, Kotaidis V, Ballot H, Plech A. Turkevich method for gold nanoparticle synthesis revisited. J Phys Chem B 2006;110:15700-7.

16. Livingston JD, dan Carpay FM. Controlled nucleation for the regulation of the particle size in monodisperse gold suspensions. Nat Pyhs Sci 1973;241:20.

17. Turkevich J. Colloidal gold, part II: Colour, coagulation, adhesion, alloying and catalytic properties. Gold Bull 1985;18:125-31.

18. Motion B. Dynamic Light Scattering : An Introduction in 30 Minutes, OLS Technical Note. p1-8

19. Malvern I. Inform White Paper Dynamic Light Scattering. Malvern: Malvern Instruments; 2011. p. 1-6.

20. Nidhin M, Indumathy M, Sreeram K, dan Nair B. Synthesis of iron oxide nanoparticles of narrow size distribution on polysaccharide templates. Bull Mater Sci 2008;31:93-6.

21. Dutta J, dan Sugunan A. Colloidal Self-Organization for Nanoelectric. Kuala Lumpur: Proceeding IEEE International Conference on Semiconductor Electronics. IEEE; 2004. p. 6.

22. Kattumuri V. Gold Nanoparticles for Biomedical Applications: Synthesis, Characterization, in vitro and in vivo Studies. Dissertation the Faculty of Graduate School. Columbia: University of Missouri; 2006. 